\title{
The microgenetic development of the Ponzo and Zöllner illusions
}

\author{
ROBERT I. REYNOLDS \\ Institute for Cognitive Studies, Rutgers University, Newark, New Jersey 07102
}

\begin{abstract}
It is argued that in order to most directly investigate perceptual processes and thereby to evaluate competing theories, one must observe the (microgenetic) development of the percept. In different experiments, variations of the Ponzo and Zöllner illusions were tachistoscopically presented, followed, after a variable ISI, by a disrupting pattern. The interaction responsible for an illusion of magnitude in the Ponzo and an illusory displacement in the Zöllner occur only after $150 \mathrm{msec}$ of processing time (SOA), Prior to the illusion's onset, the parts of the figure are clearly and accurately perceived, indicating that the parts of the figure are first processed independently, followed by the interaction of those parts. It is suggested how the application of information processing techniques to traditional perceptual material may, in future experiments, yield a positive theoretical statement about perceptual processes.
\end{abstract}

Implicit in any theory of perception is the fact that perceptual experience is the result of a sequence of events, i.e., a process. This obvious statement is all too often overlooked when, in traditional perception research, inferences are made about perceptual mechanisms based on phenomenal reports which are actually end states of the process to be explained. Inevitably, any number of different theories can and do offer explanations of the process leading to the final percept in question. It is apparent to this writer that in order to evaluate the domain of applicability of different theoretical explanations, as well as to most directly investigate the question of process, it will be necessary to chart the temporal course of the percept from the time of stimulus presentation until there is a relatively stable, wholistic perceptual experience.

The Leipzig Gestaltists conducted countless experiments investigating the development of the percept, a process which Werner (1956) referred to as "microgenesis" (for a review of this research, see Flavell \& Draguns, 1957). The results of these experiments suggested to them that the whole was not only greater than the sum of the parts, but that perception of the whole precedes perception of the parts. Unfortunately, these experiments are open to a number of methodological criticisms. They typically involved repeated presentation of the same stimulus under conditions of increasing ontime, brightness, or size. It is a real possibility that under extremely reduced stimulus conditions, the subjects expressed their uncertainty about what was presented by a simple or "pragnant" description. That is, these early experiments may not reflect subjects' perceptions as much as those conditions under which a response bias occurs (a similar criticism was advanced by Rock \&
Engelstein, 1959). It is also known that repeated presentation of the same stimulus will lead to increased performance even without altering stimulus conditions (Haber \& Hershenson, 1965). A further difficulty in interpreting the early microgenetic research is that in no case do we find a direct manipulation of processing time. (This is understandable, of course, in view of the fact that the research predates studies on iconic representation.)

Information processing techniques, which provide the potential for such controlled investigation, unfortunately have not been applied to the development of the perceptual experience. The work on masking, inhibitory mechanisms, and signal detection serve only to specify those conditions under which stimuli will or will not be seen. Thus far, there has been no investigation of qualitative changes in experience across time using these techniques.

The present research investigates the temporal development of the Ponzo and Zollner illusions using techniques similar to backward masking. More specifically, the questions of interest are: (1) How much processing time is required for perceptual organization (i.e., an illusion) to occur? (2) What is the nature of the perceptual experience prior to the illusion's onset? The problems inherent in repeated presentations are eliminated by random presentation of different versions of the illusion being investigated. The figures are presented with sufficient ontime and illumination to produce a clear iconic representation. After a variable ISI, a second figure is presented, which serves to interrupt processing of the initial target figure. Processing time is measured in terms of the stimulus onset asynchrony (SOA), which is the duration from the time the target comes on until presentation of the second, disrupting pattern. 


\section{EXPERIMENT 1}

\section{Method}

In Experiment 1, two target figures were used: the standard Ponzo (A in Figure 1) and a figure (B in Figure 1) in which the upper horizontal is physically shorter. The converging lines in both figures were $90 \mathrm{~mm}$ long, converging at an angle of $30^{\circ}$. Both horizontals in A were $27 \mathrm{~mm}$ long. In B, the top line was $22 \mathrm{~mm}$ long and the bottom $27 \mathrm{~mm}$ long. With unlimited processing time, observers' descriptions of B are equally divided between the lines being equal and the bottom line being longer. The figure presented after the target was drawn so as to coincide perfectly with the external lines of the target figure. It will be referred to as the "disrupting stimulus," or simply "DS." Comparable results are obtained whether the disrupting stimulus (1) consists of random dots appearing in the area of the horizontal inducing lines, (2) contains the inducing lines embedded within a pattern of other lines, or (3) shows the inducing lines alone. Conditions using noise pattern configurations yield psychophysical curves similar to those to be discussed, except for a much higher degree of random variation in the estimation of the horizontal test lines. The simpler DS, shown in Figure 1, serves to disrupt only the interaction between converging and horizontal lines, and was therefore selected for use in the conditions to be discussed.

The figures were presented in a three-channeled tachistoscope. Subjects observed the figures from an effective distance of $76.2 \mathrm{~cm}$. In all of the experiments to be discussed, the target figure was on for $50 \mathrm{msec}$, and the DS for $200 \mathrm{msec}$. The SOAs were $50,75,100,150,200$, and $250 \mathrm{msec}$. The interval between target and DS was filled with a blank field of the same luminance as that of the target and DS. A small dot in the center of the field was on continuously, and served as a fixation point.

By pressing a button, the subjects were able to control when the figure was presented. They were asked to fixate on the small dot in the center of the field, and, after each presentation, to describe their impressions of the relative lengths of the horizontal lines by saying "top is longer," "bottom is longer," or "equal." Unknown to the subject, the first four trials constituted practice and were not counted in the data. Following the four practice trials, the target figures, A and B, were each presented once at each of the six intervals. The order of presentation of each figureSOA combination was randomized for each subject. On completion of the experiment, subjects were asked to describe the clarity and distinctiveness of the lines in the target figure.

Fifteen students taking an introductory psychology course participated in Experiment 1 as partial fulfillment of their course requirements.

\section{Results}

Table 1 gives the number of subjects who responded "top is longer" (T), "bottom is longer" (B), or "equal" ( $=)$, at each figure-SOA combination. Figure 2 presents the number correct for both figures across SOAs. The subjects make mostly veri-
Experiment :
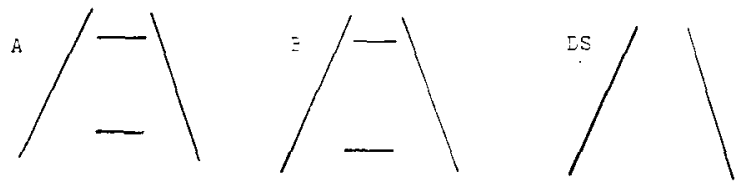

Experiment 2
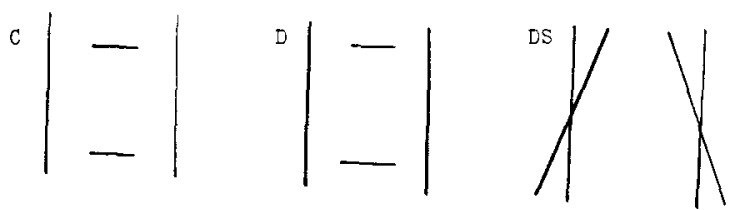

Figure 1. Stimuli used in Experiments 1 and 2.

dical judgments from SOAs of 50 to $100 \mathrm{msec}$. For both versions of the Ponzo illusion, there is a significant increase in the illusion at an SOA of $150 \mathrm{msec}$. The chi square for Figure $A$ is $23.02(p<.001)$ and for Figure $B, \chi^{2}=9.28(p<.01)$. The standard version (Figure $A$ ) does not achieve its highest value of illusion until $250 \mathrm{msec}$. These findings indicate that the converging lines of the target influence perception of the horizontal test lines only when the processing time is longer than $100 \mathrm{msec}$. It will be necessary in future experiments to investigate more precisely when, during the 100 - to 150 -msec interval, the interaction occurs.

\section{EXPERIMENT 2}

In Experiment 1, the subjects' phenomenal reports suggested that the parts of the figure-the horizontal and converging lines-appear clear and distinct from the DS at all SOAs, except for the converging lines at an ISI of 0 msec (SOA of 50). Experiment 2 was intended to quantify this finding by requiring subjects to discriminate between different orientations of external lines.

\section{Method}

In Experiment 2, four target figures were used: Figures $A$ and $B$ as in Experiment 1, and two others (see $\mathrm{C}$ and $\mathrm{D}$ in Figure 1)

Table 1

Judgment of Horizontals as "Longer" or "Equal" in Experiment 1

SOA (milliseconds)

\begin{tabular}{|c|c|c|c|c|c|c|c|c|c|c|c|c|c|c|c|c|c|c|}
\hline \multirow[b]{3}{*}{$A(T=B)$} & \multicolumn{3}{|c|}{50} & \multicolumn{3}{|c|}{75} & \multicolumn{3}{|c|}{100} & \multicolumn{3}{|c|}{150} & \multicolumn{3}{|c|}{200} & \multicolumn{3}{|c|}{250} \\
\hline & $\mathrm{T}$ & $\mathrm{B}$ & $=$ & $\mathrm{T}$ & $\vec{B}$ & $=$ & $\underline{T}$ & $\mathrm{~B}$ & $=$ & $T$ & B & $=$ & $\mathrm{T}$ & B & $=$ & $T$ & $B$ & $=$ \\
\hline & 6 & 0 & 9 & 4 & 2 & 9 & 3 & 1 & 11 & 10 & 0 & 5 & 12 & 1 & 2 & 15 & 0 & 0 \\
\hline$B(T<B)$ & 2 & 5 & 8 & 2 & 7 & 6 & 2 & 7 & 6 & 5 & 0 & 10 & 4 & 1 & 10 & 4 & 1 & 10 \\
\hline
\end{tabular}

Note $-T=$ top line appears longer; $B=$ bottom line appears longer: "=" signifies that lines appear equal. 


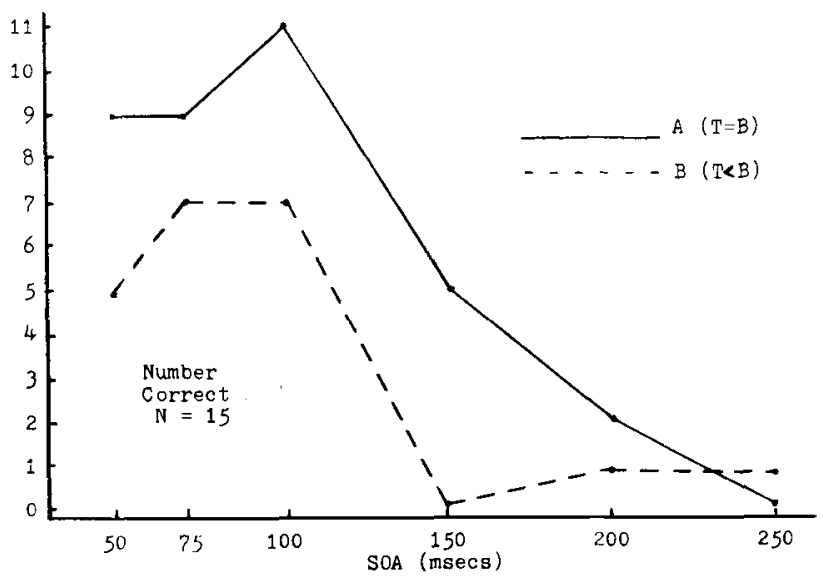

Figure 2. Number correct in Experiment 1.

which had the same length of horizontal lines but were surrounded by two vertical lines. Figures $C$ and $D$ are therefore nonillusory counterparts of Figures A and B. The DS was drawn so as to coincide with the external lines of the target figures.

Fifteen undergraduate students participated in this experiment. Subjects were instructed to report whether the external lines were converging or parallel, as well as their impression of the relative lengths of the horizontal lines. Following four practice trials, Target Figures A, B, C, and D were each presented once at each of the six intervals used in Experiment 1. The order of presentation of each figure-SOA combination was randomized for each subject.

\section{Results}

Table 2 gives the number of subjects who responded "T," "B," or "=" at each figure-SOA combination. As in Experiment 1, there is little or no illusion with Figures $A$ and $B$ until an SOA of $150 \mathrm{msec}$. The difference between SOAs of 150 and $100 \mathrm{msec}$ is statistically significant for Figure A $\left(\chi^{2}=11.6, \mathrm{p}<.01\right)$, while the difference between 150 and 75 is significant for Figure $B\left(\chi^{2}=6.16\right.$, $\mathrm{p}<.05)$. The nonillusory figures, $\mathrm{C}$ and $\mathrm{D}$, with vertical lines do not show any systematic changes across SOAs.

Table 3 gives the number of subjects who responded "converging" (C) or "parallel" (P) at each figure-SOA combination. The subjects were extremely accurate in reporting whether the external lines were converging or parallel, except at an SOA of $50 \mathrm{msec}$, when the response was at a chance level. The initial $50 \mathrm{msec}$ exposure does not provide the visual system with enough time to discriminate the target from the DS. The subjects' phenomenal report, which was given at the end of a session, confirms that all of the parts of the figure are clearly perceived after an SOA of $75 \mathrm{msec}$. We may conclude, therefore, that the parts of the figure are clearly and accurately perceived after less processing time than is required for their interaction, i.e., prior to onset of the illusion. Within this context, we may address ourselves to the classical part-whole question by say-

Table 2

Judgment of Horizontals as "Longer" or "Equal" in Experiment 2

\begin{tabular}{|c|c|c|c|c|c|c|c|c|c|c|c|c|c|c|c|c|c|c|}
\hline & & & & & & & & & SOA & illise & con & & & & & & & \\
\hline & & 50 & & & 75 & & & 100 & & & 150 & & & 200 & & & 250 & \\
\hline$A(T=B)$ & $\frac{T}{3}$ & $\frac{B}{3}$ & $=$ & $\frac{T}{3}$ & $\frac{B}{3}$ & $\frac{=}{9}$ & $\frac{T}{2}$ & $\frac{B}{2}$ & $\frac{\overline{1}}{11}$ & $\frac{\mathrm{T}}{10}$ & $\frac{\bar{B}}{0}$ & $=$ & $\frac{T}{14}$ & $\frac{B}{0}$ & $\frac{=}{1}$ & $\frac{T}{15}$ & $\frac{\vec{B}}{0}$ & $\frac{=}{0}$ \\
\hline$B \quad(T<B)$ & 0 & 8 & 7 & 2 & 7 & 6 & 3 & 6 & 6 & 4 & 1 & 10 & 4 & 0 & 11 & 4 & 0 & 11 \\
\hline$C \quad(\mathrm{~T}=\mathrm{B})$ & 2 & 4 & 9 & 3 & 2 & 10 & 2 & 3 & 10 & 3 & 1 & 11 & 0 & 2 & 13 & 0 & 0 & 15 \\
\hline$D \quad(\pi<B)$ & 0 & 8 & 7 & 1 & 8 & 6 & 2 & 7 & 6 & 0 & 7 & 8 & 1 & 8 & 6 & 0 & 8 & 7 \\
\hline
\end{tabular}

Note: $T=$ top line appears longer; $B=$ bottom line appears longer; "=" signifies that lines appear equal.

Table 3

Judgment of External Lines in Experiment 2

SOA (in milliseconds)

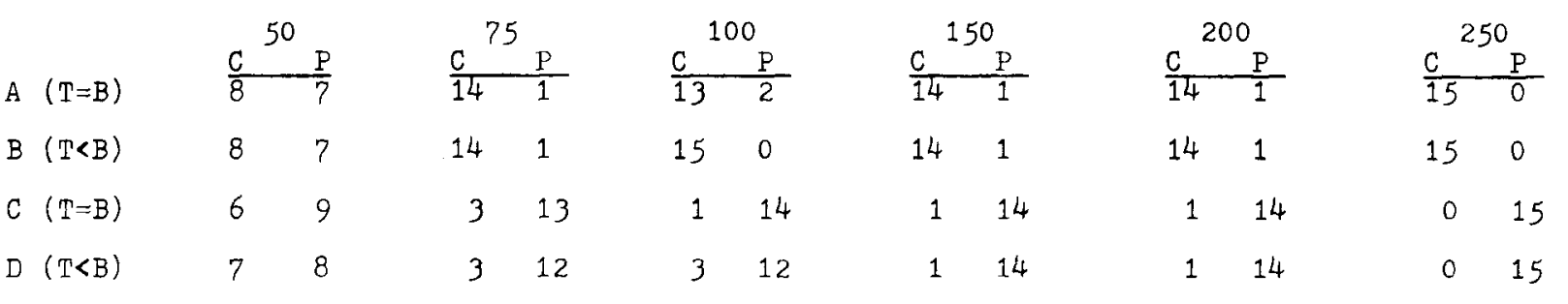


ing that perception of the parts precedes perception of the whole.

\section{EXPERIMENT 3}

Experiment 3 investigates the development of the Zöllner illusion. The Zöllner consists of two or more parallel lines, each being intersected by a number of smaller lines. The cross lines cause an illusory displacement of the parallels, such that they no longer appear parallel. Experiment 3 makes use of procedures identical to those used to investigate the Ponzo illusion.

\section{Method}

In this experiment, four target figures were used. Figure $3 \mathrm{~A}$ is one version of the Zöllner illusion, with two parallel lines drawn at an angle of $45^{\circ}$. Figure $3 B$ is a modification of the Zöllner in which the two diagonal lines physically converge toward the top. The convergence in Figure 3B leads to the illusory impression of the diagonals being parallel. Figures $3 \mathrm{C}$ and $3 \mathrm{D}$ consist of the same diagonal lines as in $\mathrm{A}$ and $\mathrm{B}$ but with horizontal cross lines intersecting both diagonals (and thus yielding no illusion). The DS consisted of two series of $+s$, drawn so as to perfectly coincide with the cross lines of the preceding target.

Fifteen students taking an introductory psychology course participated in this experiment. They were instructed to report whether the diagonal lines were "converging toward the top," "converging toward the bottom," or "parallel," as well as the relative orientation of the cross lines, i.e., whether the cross lines were going in the "same direction" or in "different directions." Following the four practice trials, Target Figures A, B, C, and D were each presented once at each of the six intervals used in the preceding experiments. The order of presentation of each figure-SOA combination was randomized for each subject.

\section{Results}

Table 4 gives the number of subjects who responded "converging toward the top" $(\mathrm{Ct})$, "converging toward the bottom"' $(\mathrm{Cb})$, or "parallel" $(\mathrm{P})$. Figure 4 presents the number correct for Figures $3 \mathrm{~A}$ and $3 \mathrm{~B}$ at each figure-SOA combination. The subjects made mostly veridical judgments at SOAs of 75 and $100 \mathrm{msec}$. The difference between SOAs of 150 and $100 \mathrm{msec}$ is statistically significant for Figure A $\left(\chi^{2}=12.18, \mathrm{p}<.003\right)$, while the difference between 150 and 75 is significant for Figure $B\left(\chi^{2}=6.0\right.$, $\mathrm{p}<.05)$. Figures $\mathrm{C}$ and $\mathrm{D}$ are described veridically at SOAs of $75 \mathrm{msec}$ or greater. "Parallel" is the predominant response to all figures at an SOA of $50 \mathrm{msec}$.

The data from Experiment 3 indicate temporal development comparable to that found in Experiments 1 and 2 with the Ponzo illusion. The interaction between cross lines and diagonals occurs again only at $150 \mathrm{msec}$ of processing time.

Table 5 gives the number of subjects who responded "same direction" (S) or "different directions" (D), in regard to the orientation of the cross lines. The subjects were accurate approximately $75 \%$ of the trials across all SOAs, except at $50 \mathrm{msec}$, when the line discrimination becomes somewhat more dif-

Table 4

Judgment of Diagonal Lines in Experiment 3

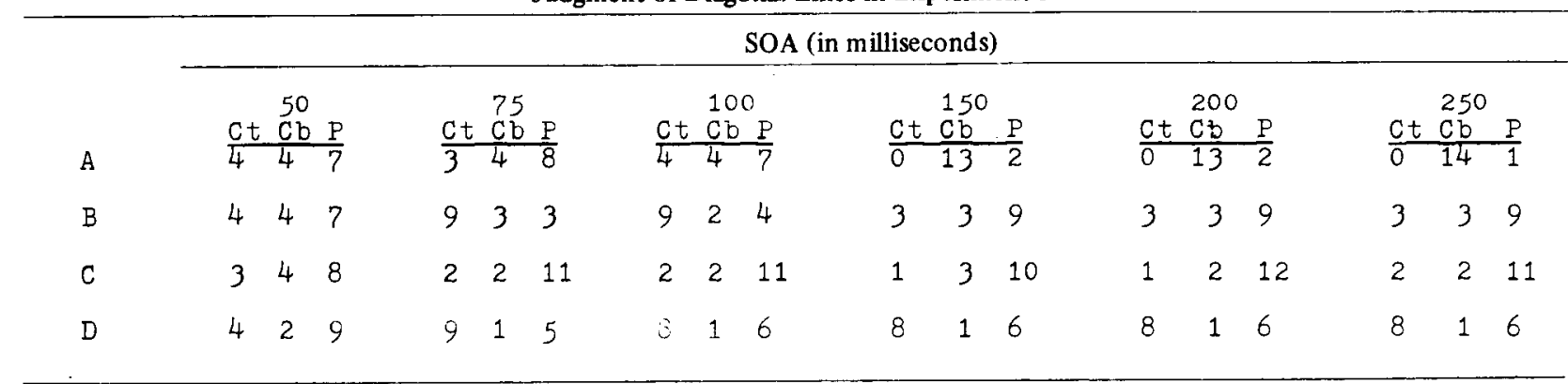

Note-Ct $=$ lines appear to converge toward the top, $C b=$ lines appear to converge toward the bottom; $P=l i n e s$ appear parallel.

Table 5

Judgment of Cross Lines in Experiment 3

\begin{tabular}{|c|c|c|c|c|c|c|c|c|c|c|c|c|}
\hline & \multicolumn{12}{|c|}{ SOA (in milliseconds) } \\
\hline & \multicolumn{2}{|c|}{50} & \multicolumn{2}{|c|}{75} & \multicolumn{2}{|c|}{100} & \multicolumn{2}{|c|}{150} & \multicolumn{2}{|c|}{200} & \multicolumn{2}{|c|}{250} \\
\hline$A(d i f f \cdot)$ & $\frac{S}{6}$ & $\frac{D}{9}$ & $\frac{\mathrm{S}}{3}$ & $\frac{D}{12}$ & $\frac{S}{2}$ & $\frac{D}{13}$ & $\frac{S}{2}$ & $\frac{D}{13}$ & $\frac{S}{2}$ & $\frac{D}{13}$ & $\frac{S}{2}$ & $\frac{D}{13}$ \\
\hline$B$ (diff.) & 6 & 9 & 5 & 10 & 5 & 10 & 3 & 12 & 3 & 12 & 2 & 13 \\
\hline $\mathrm{C}$ (same) & 8 & 7 & 10 & 5 & 9 & 6 & 11 & 4 & 10 & 5 & 11 & 4 \\
\hline D. (same) & 8 & $?$ & 9 & 6 & 10 & 5 & 9 & 6 & 10 & 5 & 11 & 4 \\
\hline
\end{tabular}


ficult (though the difference between $50 \mathrm{msec}$ and other SOAs is not statistically significant). For both Ponzo and Zöllner illusions, there is an initial $100 \mathrm{msec}$ prior to the illusion's onset during which the parts "peacefully coexist."

\section{DISCUSSION}

This research set out to investigate two basic questions: (1) What is the duration of processing time before there is an interaction between parts of a figure? (2) What is the nature of the perceptual experience prior to the interaction? In answer to the first question, virtually no illusory effects were found for either the Ponzo or Zöllner figures until $150 \mathrm{msec}$ of processing time, when there was a dramatic increase in the number of subjects reporting an illusion. In answer to the second question, discrimination tasks and the subjects' phenomenal report indicate that the parts of the figure are clearly discernible after $75 \mathrm{msec}$ of processing time. We may therefore conclude that those processes leading to perception of the parts occur after less processing time than is required for interaction of those parts. It thus appears that the contrary findings of the early microgenetic research were the result of its specific methodology and lack of controls.

The type of disrupting stimulus used serves to disrupt the interaction between parts of the figure without preventing the observer from comparing the two test lines. It is questionable whether this disruption may be classified as "backward masking." Masking generally refers to the obscuring or obliteration of a target stimulus by a preceding or subsequent stimulus (for a review of the different types of masking, see Kahneman, 1968), whereas the DS used in the present research has the effect of disrupting only the interaction.

I have applied these techniques to other stimuli with comparable results. In one set of experiments, a subjective contour pattern was presented which led with unlimited processing time to perception of a subjective triangle. After a variable ISI, a disrupting
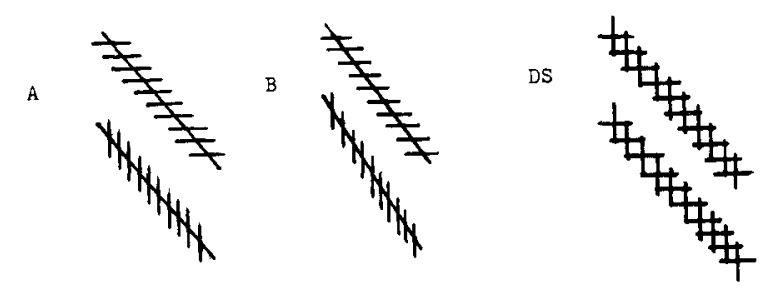

C

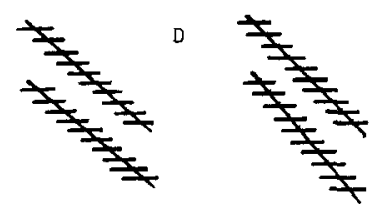

Figure 3. Stimuli used in Experiment 3.

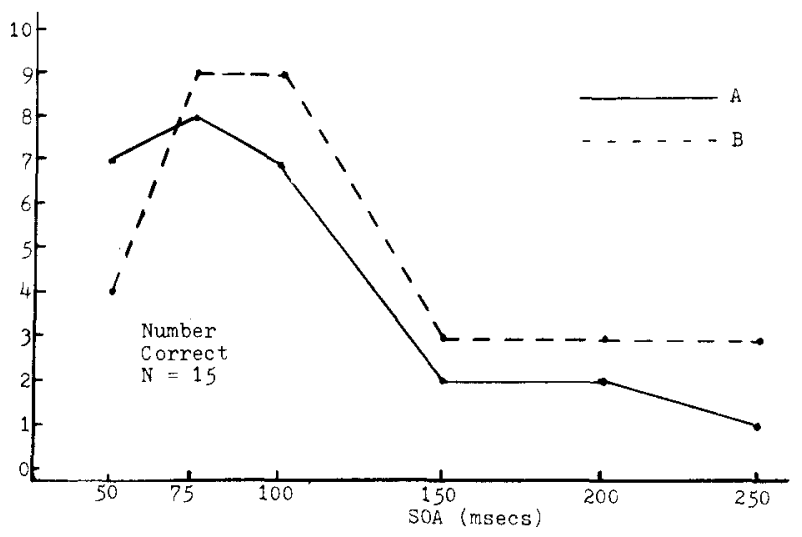

Figure 4. Number correct in Experiment 3.

pattern was presented. Findings from that series of experiments indicate that formation of a subjective contour requires somewhat less processing time than do the interactions involved in the Ponzo and Zöllner illusions-approximately $100 \mathrm{msec}$ before a contour is reported.

Similar results have been obtained using the rod and frame as stimuli. In this case, information processing techniques allow us to determine when, during processing, field dependence becomes a determining factor for some subjects. Results from a pilot study suggest that the tilt of the frame affects the perceived orientation of the rod only after $100 \mathrm{msec}$ of processing time, and then only for field-dependent subjects. In this study, the orientation of the rod was manipulated, thereby making it posible to measure the magnitude of the illusion, something that was not done in previous experiments.

These experiments do not indicate the nature of the interaction between test and inducing lines in illusion figures, except to place certain limitations on theoretical proposals. The findings preclude any theory which requires an interaction within the first $100 \mathrm{msec}$, or after approximately $200 \mathrm{msec}$ of processing time. A developmental approach has the potential not only to help determine the applicability of different theories, but to indicate the level of the nervous system responsible for the interactions leading to a visual illusion. What is needed is human neurophysiological research which charts the time sequence of a stimulus relative to its locus of processing.

I believe it is possible, in future experiments, to make a more positive theoretical statement than I have done here. This could be done by manipulating structural features of the stimulus while observing when, during one's processing of those features, different sources of information come into play or are taken into account. We may find that we are not dealing with a simple two-stage model in which the parts are first processed independently, followed by an interaction of those parts. Rather, a series of interactions may occur, each interaction or resulting structure building upon the preceding one. 


\section{REFERENCES}

Flavell, J. H., \& Draguns, J. A microgenetic approach to perception and thought. Psychological Bulletin, 1957, 54, 197.217.

Haber, R. N., \& Hershenson, M. The effects of repeated brief exposures on the growth of a percept. Joumal of Experimental Psychology, 1965, 69, 40.46.

KahNEman, D. Method, findings and theory in studies of visual masking. Psychological Bulletin, 1968, 70, 404-425.
Rock, I., \& Engelstein, P. A study of memory for visual form. American Joumal of Psychology, 1959, 72, 221-229.

Werner, H. Microgenesis and aphasia. Journal of Abnormal Social Psychology, 1956, 52, 347-353.

(Received for publication July 22, 1977; revision accepted January 4, 1978.) 